\title{
Investigation of a Community Outbreak of Diarrhea Associated with Drinking Water in Suburb of Chengdu, China
}

\author{
Wei Zeng, Shan Jiang, Xian Liang, Jun Chen, Qi Peng, Zhijun Li, Fengman Dou* \\ Chengdu Center for Disease Control and Prevention, Chengdu, China \\ Email: zengwei0623@126.com, alice712@163.com, Liangxian0325@126.com, pengqi1001@sina.com, \\ fmdou@yahoo.com.cn
}

Received 9 December 2014; accepted 4 July 2015; published 7 July 2015

Copyright $(2015$ by authors and Scientific Research Publishing Inc.

This work is licensed under the Creative Commons Attribution International License (CC BY). http://creativecommons.org/licenses/by/4.0/

(c) (i) Open Access

\begin{abstract}
Objectives: To identify the etiology and source after recognition of diarrhea outbreak is associated with drinking water in suburb of Chengdu. Methods: Both unmatched case control and retrospective cohort study were conducted. 131 targets including 56 suspected patients were recruited for case control study, while 463 residents were selected for cohort study. Stool, water and environmental samples were collected for laboratory testing. Results: The proportion of case exposed to well water was $86 \%$ in case group compared with $51 \%$ in the controls during the epidemic period $(O R=6.14, P<0.01)$. Exposing to well water $(R R=3.14, P<0.01)$ and the distance between well and river $(\mathrm{RR}=2.59, P<0.01)$ were at higher risk for diarrheal illness. Nucleic acid of Enteropathogenic $E$. coli and Enterotoxigenic $E$. coli was positive in stool and river samples. Conclusions: This outbreak of diarrhea might be caused by several mixed opportunistic pathogens in well water contaminated from the river water.
\end{abstract}

\section{Keywords}

Diarrhea, Water Contamination, Outbreak, Investigation

\section{Introduction}

The sanitary safety of drinking water has been of great concern in recent years [1] [2]. There have been many cases of public health issues caused by consumption of unsafe drinking water [3]. In United Sates, it was reported that of 28 waterborne disease outbreaks, 20 were related to drinking water in 14 states during 2005-2006

\footnotetext{
${ }^{*}$ Corresponding author.
}

How to cite this paper: Zeng, W., Jiang, S., Liang, X., Chen, J., Peng, Q., Li, Z.J. and Dou, F.M. (2015) Investigation of a Community Outbreak of Diarrhea Associated with Drinking Water in Suburb of Chengdu, China. Open Journal of Epidemiology, 5, 147-154. http://dx.doi.org/10.4236/ojepi.2015.53019 
[4]. There were 271 drinking water contamination incidents around China except Tibet autonomous region, of which 63\% were classified as biological during 1996-2006. More than 7 million people were affected [5]. Furthermore, $57 \%$ of events related to drinking water contamination were attributed to water source pollution over past 20 years in China. It is also reported only $30 \%$ of drinking water source meet the national standards due to environmental pollution, $82 \%$ of rivers and lakes were polluted to various extents in China [6].

Diarrhea is one of the most common public health problem caused by polluted water [7]. Epidemiologic reports indicate that enterotoxigenic E. coli (ETEC), Campylobacter jejuni, and Shigella spp (particularly S. flexneri and S. sonnei) are the most common causes of diarrheal disease among adults and children. In recent years, norovirus, the third most frequent pathogen, has caused several community diarrhea outbreak via drinking water [8].

Most of diarrheal diseases can be prevented by adequate management of drinking water in developing countries [9]. Rural and suburb areas of China suffered from dispersion of drinking water pollution in recent years. Widespread microbial contamination of drinking water, which possibly due to lack of water source protection and tap water supply system, dumping rubbish to the water directly, etc, increased the risk of water born disease transmission among local residents [6]. Moreover, polluted foods and fruits sold in the market also affected families with qualified tap water supply. This report described an outbreak of diarrhea associated with drinking water in a small town with mixed types of drinking water supply in China, June 2013.

\section{Methods}

\subsection{Study Site}

G town (longitude $103^{\circ} 86^{\prime}$ and latitude $30^{\circ} 65^{\prime}$ ) located at the suburb of Chengdu, Sichuan province, southwest of China, with an area of 3.62 square kilometers. There are 5522 residents, most of them work in factories nearby and live in tenements. There is one main street along the riverside, where most of the old houses and market are built. Vegetables and fruits sold in the market place are always washed with water from the river or well. There is one dump near river with daily cleaning.

Benefited from urbanization, qualified tap water supply are available in new buildings in $\mathrm{G}$ town, while most of old houses still use separate wells (5 - 18 meters deep) at their own yard. Two brands of bottled water were also provided to the local residents in two shops.

There are one Community Health Service Center (CHSC) and several private clinics in G town. According to the medical records from the CHSC, in May of 2013, there were 18 diarrhea patients in total, with no more than 3 cases per day. From $10^{\text {th }}$ to $13^{\text {th }}$, June 2013 , there were more than 30 suspected diarrhea patients, which were reported timely from doctors in CHSC to the local Center for Disease Control and Prevention (CDC) on $14^{\text {th }}$ June. The field survey aimed to investigate and control the outbreak was conducted by epidemiologists and microbiologists from Chengdu CDC and Sichuan University.

\subsection{Epidemiologic Investigations}

Suspected diarrhea cases was defined as local resident of G town who developed the following symptoms: diarrhea ( $\geq 3$ times per day) with at least one of the following symptoms: fever $\left(\geq 37.5^{\circ} \mathrm{C}\right)$, vomiting, or abdominal pain between $7^{\text {th }}$ and $21^{\text {th }}$ of June 2013. Eligible cases were identified by medical records review.

The medical information of 97 cases was from CHSC. The data collection tool based on the family unit was developed to collect following information: number of suspected cases, name, gender, address, date of onset, diarrhea frequency (per $24 \mathrm{~h}$ ), vomiting, abdominal pain, fever, type of water used, eating out and source of vegetables and fruits. The investigation team was responsible for investigation and detection of suspected cases treated in private clinics or absence of medical help. By case-finding in district with the highest diarrheal prevalence, totally, there were 156 suspected cases enrolled.

For the unmatched case control study, 59 diarrhea patients diagnosed by CHSC could be contacted were incase group and participated in face to face interview with questionnaire. 72 people, who were neighbors of the suspected cases without diarrhea symptoms between $7^{\text {th }}$ and $21^{\text {th }}$ of Jun 2013, were assigned to control group.

The retrospective cohort study was conducted in district with the highest diarrheal prevalence, which was calculated from the geographic distribution of suspected cases. 463 targets from 99 families were investigated to collect diarrheal illness information and the possible exposure including type of water use, situation of the well, 
and source of vegetables and fruits, etc.

\subsection{Specimen Collection and Laboratory Testing}

A total of 29 well water, 4 river water, 4 tap water, 3 bottled water and 33 other environmental samples (kitchen knife, vegetables, fruits, and sewer) from patients' house were collected. Physical and chemical examinations as well as pathogenic microbes test were conducted by Chengdu CDC. 8 stool samples from suspected patients were collected by local hospital for rapid detection of Vibrio cholera, therein 5 stool samples were collected for pathogenic microbes test between $7^{\text {th }}$ and $21^{\text {th }}$ by Chengdu CDC.

Quality assessment of water samples was performed on accordance with standard method [10]. Briefly, total aerobic bacterial count with standard Pour Plate technique was applied for processed water samples. Presence of coliforms and Enteropathogenic E. coli (EPEC), ETEC by Most Probable Number (MPN) and membrane filtration methods was determined. Environmental samples were homogenized under aseptic conditions. Three 10 -fold serial dilutions were prepared from homogenates to inoculate different culture media. Samples were inoculated on Salmonella Shigella (SS), Xylene lactose decarboxylase (XLD) and Campylobacter selective media.

Stool samples were analyzed microscopically for the presence of ova or parasite(s). Bacteriological analysis was performed for the detection of Salmonella, Shigella, E. coli, Vibrio cholera with MacConkey's agar, SS agar, TCBS agar and Sorbitol Mac-Conkey's agar (Oxoid). Half pea sized samples were inoculated on culture media plates and incubated aerobically at $37^{\circ} \mathrm{C}$ for 48 hours. Transport swabs were further immersed in Selenite F broth (Oxoid).

PCR testing was conducted for the following virulence makers or bacteria: Enteropathogenic E. coli (EPEC), ETEC, Shigella spp, Salmonella, Staphylococcus aureus, Vibrio parahemolyticus. Bacterial isolates from environmental and clinical samples were processed for identification with standard biochemical reactions such as oxidase, triple sugar iron, indole, sulfide, motility, citrate and urea hydrolysis. API20E strips were used for further confirmation. Real-time PCR testing was conducted to detect Norovirus and Rotavirus.

\subsection{Statistical Analysis}

All statistical analyses were performed by SAS (version 9.0). Student's t test and the Persons chi-square testwas applied to examine the differences of demographic characteristics between cases and controls. Odds ratio (OR), relative risk (RR) and 95\% confidence intervals (CIs) of risk factors and possible interaction were assessed with multivariate logistic regression in both case-control and cohort design. $P$-values less than 0.05 were considered to be statistically significant.

\subsection{Ethical Considerations}

The ethics committee of Chengdu CDC waived the need for informed consent for this study because it was conducted to fulfill public health mandate rather than human subject research. None of authors were involved in sample collection. All samples and information from suspected patients were anonymous and voluntary. There is no specific permissions required for this investigation in this place.

\section{Results}

\subsection{Epidemiological Investigation}

There were totally 156 eligible cases enrolled. In June of 2013, the epidemic curve showed an increased number of diarrhea cases from $7^{\text {th }}$ to $21^{\text {st }}$ (Figure 1). There were 20, 24, 19 and 22 suspected cases per day during $11^{\text {st }}$ to $14^{\text {th }}$ respectively, with a peak in curve on $12^{\text {nd }}$. The number of new reported cases declined after $16^{\text {th }}$, when residents stopped to use the well water. The last case of this outbreak was reported on $21^{\text {st }}$. Accordingly, the outbreak could be linked to a continuous common source by the characteristics as slow beginning, explosive increasing of cases in short time.

Among the 97 suspected cases that went to the CHSC for treatment, 42\% (41/97) were male. The median age was 36 years old (range: 10 to 65 years). Common symptoms reported including dehydration (79\%), abdominal pain (59\%), nausea (43\%), vomiting (30\%), headache (4\%) and fever (1\%). According to the cases self-report, the median times of diarrhea before seeking doctor were 5 times per day (range: 3 to 12 times). 91\% (88/97) and 
9\% (9/97) cases reported watery stool and loose stool respectively. 91\% (88/97) cases had taken antibiotics before seeking doctor. 63\% (61/97) of cases were treated in hospital with Amoxicillin and Clarithromycin (Table 1).

59 cases found by investigation team were from 36 families. There were 18 families had more than one patient, while a maximum of 4 cases gathered in one family. The strong household clustering of cases ( $61 \%$ interviewed families had more than one case) increased the possibility of common exposure or contagious of the disease. The type of water used included well water (78\%), bottled water (61\%) and tap water (39\%), while parts of families used more than one type of water. 31\% (11/36) families had history of dining out in local restaurant three days before diarrhea onset, yet all investigated families deny dining outside in the same restaurant. Additionally, 42\% (15/36) families bought vegetables and fruits on the street market.

\subsection{Case Control Study}

The mean age of cases and controls were $36.92(\mathrm{SD}=13.12)$ and 40.10 years old $(\mathrm{SD}=16.14)$, respectively $(\mathrm{t}=$ $1.220, P=0.23)$. There were 33 males and 26 females in case-group, 35 males and 37 females in control-group $\left(\chi^{2}=0.696, P=0.40\right)$. Diarrhea was statistically significant associated with well water exposure during $7^{\text {th }}$ to

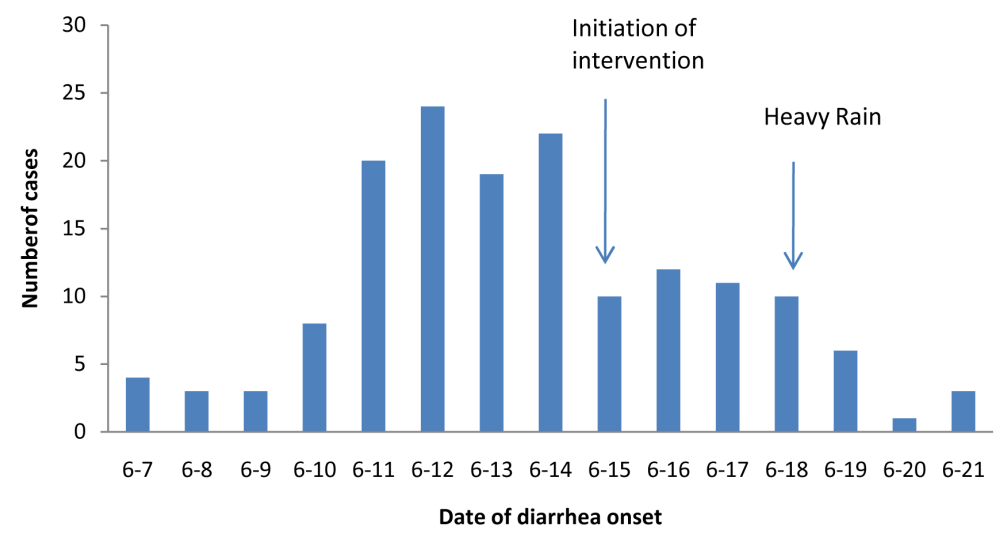

Figure 1. Epidmic curve of cases with diarrhea in $\mathrm{G}$ town, Chengdu.

Table 1. Demographic and Clinical Characteristic of 97 suspected cases seeking treatment in the CHSC.

\begin{tabular}{ccc}
\hline Demographic and Clinical Characteristics & Overall (n=97) & $\%$ \\
Age -years & 36 & Range: $10-65$ \\
Gender -male & 41 & 43 \\
Dehydration & 77 & 79 \\
Abdominal pain & 57 & 59 \\
Nausea & 42 & 43 \\
Vomiting & 29 & 30 \\
Headache & 4 & 4 \\
Fever & 1 & Range: $3-12$ \\
Times of diarrhea before seeing a doctor & 5 & 91 \\
Water stool & 88 & 9 \\
Loose stool & 9 & 98 \\
\hline
\end{tabular}


$21^{\text {th }}$ of June (OR $\left.=6.03,95 \% \mathrm{CI}: 2.34-16.0, P<0.01\right)$. Parts of residents who use the tap water only developed diarrheal symptom in case control and cohort settings (the incidence is $7 \%$ ), however, the statistically significant was not observed between variables including gender, bottled water, tap water, dining out and bought vegetables and fruits in local market and diarrhea. (Table 2)

A multivariate logistic regression analysis indicated that well water exposure during $7^{\text {th }}$ to $21^{\text {st }}$ June was associated with increased risk of diarrhea $(\mathrm{OR}=6.14,95 \% \mathrm{CI}$ : $2.46-18.2, P<0.01)$, while tap water exposure was considered as protective factor $(\mathrm{OR}=0.36,95 \% \mathrm{CI}: 0.18-0.74, P=0.04)$. (Table 2$)$

\subsection{Retrospective Cohort Study}

Among 463 cases from 99 families, there were $16 \%$ (76/463) cases had the diarrheal symptom during $7^{\text {th }}$ to $21^{\text {st }}$ June. The cohort study indicated that well water exposure was at higher risk for diarrheal illness $(\mathrm{RR}=3.14$, 95\%CI: $1.78-5.53, P<0.01)$. In addition, the distance between the well and river $(<30 \mathrm{~m})$ was statistically associated with diarrheal illness ( $\mathrm{RR}=2.59,95 \% \mathrm{CI}$ : $0.94-7.14, P=0.04)$ in families with wells. In contrary, exposed to tap water (RR $=0.32$, 95\%CI: $0.18-0.56, P<0.01$ ) was considered to prevent people from diarrhea illness. The use of bottled water was not statistically associated with risk of diarrheal illness. (Table 3 )

\subsection{Laboratory Results}

33 environmental, 3 bottled water and 4 tap water samples were negative for pathogenic bacteria. Coliform bacteria was found in 2 of 4 river water samples and total numbers of colony and bacterium was more than 100 $\mathrm{CFU} / \mathrm{ml}$ in 2 river water sample. Nucleic acid of EPEC and ETEC was detected in 1 sample, and ETEC (O128: K67 (B12)) was isolated from 1 sample. Sobria was detected in 1 of 29 well water samples.

Vibrio cholera was negative in 8 stool samples in rapid test. PCR testing was performed for 5 stool samples pathogenic bacteria. Pathogens were identified in 3 samples and nucleic acid of EPEC was found in 2 samples,

Table 2. Logistic regression analysis of risk factors of diarrheal illness for Control Case Study.

\begin{tabular}{|c|c|c|c|c|c|c|c|c|}
\hline & \multicolumn{2}{|c|}{$\mathrm{N}$} & \multicolumn{2}{|c|}{$\%$} & \multicolumn{2}{|l|}{ Unadjusted } & \multicolumn{2}{|l|}{ Adjusted } \\
\hline & Cases & Controls & Cases & Controls & OR ( $95 \%$ CI ) & $\boldsymbol{P}$ value & OR ( 95\%CI ) & $\boldsymbol{P}$ value \\
\hline Female & 26 & 37 & 44 & 51 & $0.75(0.37-1.49)$ & 0.40 & & \\
\hline Well water & 51 & 37 & 86 & 52 & $6.03(2.34-16.0)$ & 0.01 & $6.14(2.46-18.2)$ & 0.01 \\
\hline Bottled water & 40 & 38 & 68 & 53 & $1.88(0.87-4.11)$ & 0.08 & & \\
\hline Tap water & 20 & 34 & 34 & 47 & $0.57(0.26-1.24)$ & 0.12 & $0.36(0.18-0.74)$ & 0.04 \\
\hline Eating out & 18 & 24 & 31 & 33 & $0.88(1.42-1.84)$ & 0.73 & & \\
\hline Buy food in local market & 38 & 41 & 64 & 57 & $1.37(0.67-2.78)$ & 0.39 & & \\
\hline
\end{tabular}

Table 3. Logistic regression analysis of risk factors of diarrheal illness for Retrospective Cohort Study ( $\mathrm{N}=463)$.

\begin{tabular}{|c|c|c|c|c|}
\hline Type of water supply & Yes/No & Diarrhea & RR (95\% CI) & $\boldsymbol{P}$ value \\
\hline \multirow{3}{*}{ Well water } & Yes & $63(22 \%)$ & \multirow{3}{*}{$3.14(1.78-5.53)$} & \multirow{3}{*}{0.00} \\
\hline & & & & \\
\hline & No & $13(7 \%)$ & & \\
\hline \multirow{3}{*}{ Bottle water } & Yes & 48 (19\%) & \multirow{3}{*}{$1.35(0.880-2.072)$} & \multirow{3}{*}{0.17} \\
\hline & & & & \\
\hline & No & 28 (14\%) & & \\
\hline \multirow{3}{*}{ Tap water } & Yes & $13(7 \%)$ & \multirow{3}{*}{$0.32(0.18-0.56)$} & \multirow{3}{*}{0.00} \\
\hline & & & & \\
\hline & No & $63(22 \%)$ & & \\
\hline \multirow{2}{*}{$\begin{array}{l}\text { Distance between well and } \\
\text { river }(<30 \mathrm{~m})\end{array}$} & Yes & 17 (27\%) & \multirow{2}{*}{$2.59(0.94-7.14)$} & \multirow{2}{*}{0.04} \\
\hline & No & $4(10 \%)$ & & \\
\hline
\end{tabular}


ETEC (O7: K1 (L)) was isolated from 1 sample. Shigella spp, Salmonella, Staphylococcus aureus, Vibrio parahemolyticus, Norovirus and Rotavirus were not detected in 5 stool samples.

\section{Discussion}

Based on the clinical symptoms, epidemiological and environmental investigation findings, as well as laboratory test results, we concluded it might be well water contamination with several mixed opportunistic pathogens from the river water caused the outbreak diarrhea.

In long period, the river was polluted by rubbish dumping, hyper-sediment concentration, and severe deposition of the channel in $\mathrm{G}$ town. Due to the continuous high temperature in June, without rain and shutdown of the dam, the bacterial cloud multiplied rapidly in the river. The laboratory test of river water also proved the river water was heavily polluted. Moreover, the average depth of well along the river is 12 meters. Accordingly, the polluted river water could be easily permeated into the well. Moreover, vegetable and fruits in the market was washed by water from river, which enabled a large scale outbreak rather than limited residents exposed to contaminated well water. This point was accordance with parts of residents with diarrheal symptom but use the tap water only.

E. coli could be possible factors of the household clustering since the multiple routes of transmission, such as food and items besides water [11] [12]. Whereas, 33 environmental samples, including kitchen knife, vegetables, fruits and sewer from patients' family were all negative for pathogenic bacteria, the reason might be the latency (after $16^{\text {th }}$ Jun.) of environmental samples collection. Besides, interventions including health education, notification for temporarily suspend of water from river and well directly, etc. was commenced since $15^{\text {th }}$ Jun could contribute to the negative results.

After the heavy rain on $18^{\text {th }}$ June and river refreshing by dam opening, local people continue to use the well water as usual. Surveillance data revealed that prevalence of diarrhea fail down to normal among local residents. The clinical symptoms observed including diarrhea and dehydration at primary stage, abdominal pain, and few cases reported headache or fever in the outbreak. These clinical features closely resembled bacterial etiology [8]. The laboratory test identified several bacteria, mainly E. coli, from stool, water of well and river samples. Due to most cases $(91 \%)$ had antibiotic treatment before diagnosed. It was hard to identify pathogenicbacteria in stool. But the polymicrobial findings support the assumption that heavy contamination of river water may played a main role. The limitations in pathogen identification highlight the importance of field epidemiology.

The new reported cases of diarrhea on $16^{\text {th }}$ Jun. obviously declined, one day after intervention launched, and fell back to normal levels in 6 days, which implicated the action was timely and effective. There are two worth noting points throughout the whole epidemic course. Early detection of the outbreak with the sensitive disease surveillance system was the first point, while doctor in CHSC informed the local CDC when they noticed the abnormal increase of diarrhea cases. Secondly, with guidance on emergency response mechanism, local CDC staff conducted appropriate and field epidemiological investigation in time. Based on preliminary founding, drinking water contamination was suspected as main cause of outbreak. Targeted actions were initiated immediately, including disinfection at houses of diarrhea cases, temporarily close well water, requirement towards farmers to stop washing vegetable and fruit with water from well and river, refreshing river by opening the dam, as well as health education. Accordingly, an effective disease surveillance system and emergency response mechanism are of great importance for the early detection and recognition of similar outbreaks as well as promptly epidemic control [13] [14].

Besides waterborne transmission, other routes of transmission such as fecal-oral may also exist. The number of reported cases probably underestimates actual infection, as the clinical symptoms of diarrhea caused by $E$. coli is usually mild and self-limited, which decreased the number of patients go to hospital. In this study, no cases under 10 years of age was found, the possible reason is that children preferred to see a doctor in children's hospital instead of CHSC, and most of children eat at the school with tap water.

WHO recommends determining an outbreak, 15 - 20 proper clinical specimens need to collect. We only took 13 stool specimens for laboratory tests in this study. The lack of sufficient number of specimens taken during this investigation might have hindered the identification of the responsible pathogen in the outbreak. Moreover, it is hard to detect the pathogenic bacteria in the stool samples due to most diarrhea cases had taken antibiotic treatment by themselves before go to hospital. In addition, diarrhea is a common disease in this season, so parts of the cases may not be caused by the contaminated water. Therefore, it is necessary to control report bias in the 
future as to ensure rapid and targeted response towards outbreaks of diarrhea. However, it is worth noting that it is hard to identify the responsible pathogen in many outbreak of diarrhea for various reasons in developing counties, field epidemiological investigation is the most effective way to find risk factors and take effective control measures.

\section{Conclusion}

The outbreak of diarrhea might be caused by well water contaminated by several mixed opportunistic pathogens from the river water. Temporarily close well water and refreshing the contaminated river were highly effective to epidemic control. It is believed that to shut down well and construct centralized water supply system could be essential interventions towards waterborne diarrhea in area without tap water supply.

\section{Acknowledgements}

The authors are grateful to the staffs participated in the field investigation and interview from Wenjiang CDC, Shuangliu CDC and Tianfu CHSC in Chengdu. The authors would also like to appreciate staffs from national field epidemiology training program of China CDC for their assistance during this investigation.

\section{Author Contributions}

WZ, SJ, FMD, JC, CH, LX and MW participated in the design of this study, WZ, SJ, JC, CH, MW, QP and ZCL conducted the field survey, WZ, SJ, HC and MW performed the statistical analysis, WZ and SJ drafted the manuscript.

\section{References}

[1] Dale, K., Kirk, M. and Sinclair, M. (2010) Reported Waterborne Outbreaks of Gastrointestinal Disease in Australia Are Predominantly Associated with recreational Exposure. Australian and New Zealand Journal of Public Health, 34, 527-530. http://dx.doi.org/10.1111/j.1753-6405.2010.00602.x

[2] Howard, C.M., Handzel, T. and Hill, V.R. (2010) Novel Risk Factors Associated with Hepatitis E Virus Infection in a Large Outbreak in Northern Uganda: Results from a Case-Control Study and Environmental Analysis. The American Journal of Tropical Medicine and Hygiene, 83, 1170-1173. http://dx.doi.org/10.4269/ajtmh.2010.10-0384

[3] Farooqui, A., Khan, A. and Kazmi, S.U. (2009) Investigation of a Community Outbreak of Typhoid Fever Associated with Drinking Water. BMC Public Health, 9, 476. http://dx.doi.org/10.1186/1471-2458-9-476

[4] Yoder, J., Roberts, V. and Craun, G.F. (2008) Surveillance for Waterborne Disease and Outbreaks Associated with Drinking Water and Water Not Intended for Drinking-United States, 2005-2006. MMWR Surveillance Summaries, 57, 1105.

[5] Wang, Q., Zhao, Y.-C. and Qu, W.-D. (2010) Investigation of Drinking Water Contamination Incidents in China during 1996-2006. Journal of Environmental Health, 27, 328-331.

[6] Li, L.J., Liang, L.Q. and Liu, C.M. (2007) Analysis and Strategy of Drinking Water Pollution Accidents in Recent 20 Year s in China. Acta Geographica Sinica, 62, 917-924.

[7] Kasper, M.R., Lescano, A.G. and Lucas, C. (2012) Diarrhea Outbreak during U.S. Military Training in El Salvador. PLOS ONE, 7, e40404. http://dx.doi.org/10.1371/journal.pone.0040404

[8] Yang, Z.C., Wu, X.W. and Li, T.G. (2011) Epidemiological Survey and Analysis on an Outbreak of Gastroenteritis Due to Water Contamination. Biomedical and Environmental Sciences, 24, 275-283.

[9] World Health Organization (2005) Guidelines for the Control of Shigellosis, Including Epidemics Due to Shigella dysenteriae Type 1. http://www.who.int/topics/cholera/publications/shigellosis/en/

[10] World health Organization (2006) From Microbial Impact. Guidelines for Drinking Water Quality Incorporating First Addendum. http://www.who.int/water_sanitation_health/dwq/gdwq0506.pdf

[11] Hart, C.A., Batt, R.M. and Saunders, J.R. (1993) Diarrhoea Caused by Escherichia coli. Annals of Tropical Paediatrics, 13, 121-131.

[12] Dale, K., Kirk, M. and Sinclair, M. (2010) Reported Waterborne Outbreaks of Gastrointestinal Disease in Australia Are Predominantly Associated with Recreational Exposure. Australian and New Zealand Journal of Public Health, 34, 527-530. http://dx.doi.org/10.1111/j.1753-6405.2010.00602.x

[13] Beaudeau, P., de Valk, H. and Vaillant, V. (2008) Lessons Learned from Ten Investigations of Waterborne Gastroente- 
ritis Outbreaks, France, 1998-2006. Journal of Water and Health, 6, 491-503. http://dx.doi.org/10.2166/wh.2008.051

[14] Ranjbar, R., Rahbar, M. and Naghoni, A. (2011) A Cholera Outbreak Associated with Drinking Contaminated Well Water. Archives of Iranian Medicine, 14, 339-340. 